\title{
Geometric and Analytic Connections of the Jensen and Hermite-Hadamard Inequality
}

\author{
Zlatko Pavić*
}

(Communicated by Nihal YILMAZ ÖZGÜR)

\begin{abstract}
The aim of this paper is to present connections between the Jensen and Hermite-Hadamard inequality. The study includes convex functions of one and several variables. The basis of the research are convex combinations with the common center.
\end{abstract}

Keywords: convex combination; convex function; simplex; Jensen's inequality.

AMS Subject Classification (2010): Primary: 26B25; Secondary: 52A40; $52 B 11$.

${ }^{*}$ Corresponding author

\section{Introduction}

In summary form, we present the concept of convexity and affinity by using binomial combinations.

Let $\mathcal{X}$ be a real linear space. Let $a, b \in \mathcal{X}$ be points and let $\alpha, \beta \in \mathbb{R}$ be coefficients. Their binomial combination $\alpha a+\beta b$ is convex if $\alpha, \beta \geq 0$ and if $\alpha+\beta=1$. If $c=\alpha a+\beta b$, then the point $c$ itself is called the combination center.

A subset of $\mathcal{X}$ is convex if it contains all binomial convex combinations of its points. The convex hull conv $\mathcal{S}$ of a set $\mathcal{S} \subseteq \mathcal{X}$ is the smallest convex set which contains $\mathcal{S}$, and it consists of all binomial convex combinations of the points of $\mathcal{S}$.

Let $\mathcal{C} \subseteq \mathcal{X}$ be a convex set. A function $f: \mathcal{C} \rightarrow \mathbb{R}$ is convex if the inequality

$$
f(\alpha a+\beta b) \leq \alpha f(a)+\beta f(b)
$$

holds for all binomial convex combinations $\alpha a+\beta b$ of pairs of points $a, b \in \mathcal{C}$.

Using the adjective affine instead of convex, requiring the coefficients condition $\alpha+\beta=1$, and requiring the equality in equation (1.1), we get a characterization of the affinity.

Implementing mathematical induction, it can be verified that the above concept applies to $n$-membered affine or convex combinations for any positive integer $n>2$.

We present the discrete and integral form of the famous Jensen's inequality using convex and measurable sets.

In 1905, applying mathematical induction to convex combinations, Jensen (see [6]) has obtained the following discrete inequality.

Discrete form of the Jensen inequality. Let $\mathcal{C}$ be a convex set of a real linear space, and let $\sum_{i=1}^{n} \alpha_{i} a_{i}$ be a convex combination of points $a_{i} \in \mathcal{C}$.

Then every convex function $f: \mathcal{C} \rightarrow \mathbb{R}$ satisfies the inequality

$$
f\left(\sum_{i=1}^{n} \alpha_{i} a_{i}\right) \leq \sum_{i=1}^{n} \alpha_{i} f\left(a_{i}\right) .
$$

Received : 16-February-2015, Accepted : 09-February-2016 
In 1906, working on transition to integrals, Jensen (see [7]) has stated the another form.

Integral form of the Jensen inequality. Let $\mathcal{I} \subseteq \mathbb{R}$ be an interval. Let $\mathcal{A}$ be a measurable set of a space of positive measure $\mu$ so that $\mu(\mathcal{A})>0$, and let $g: \mathcal{A} \rightarrow \mathbb{R}$ be an integrable function so that $g(\mathcal{A}) \subseteq \mathcal{I}$.

Then every convex function $f: \mathcal{I} \rightarrow \mathbb{R}$, such that $f(g)$ is integrable, satisfies the inequality

$$
f\left(\frac{1}{\mu(\mathcal{A})} \int_{\mathcal{A}} g(x) d \mu\right) \leq \frac{1}{\mu(\mathcal{A})} \int_{\mathcal{A}} f(g(x)) d \mu .
$$

Because of its attractiveness, the Jensen and related inequalities were studied during the whole last century. So, there are the Steffensen, Brunk and Olkin inequality. In this century the research goes on and we got the Jensen-Mercer and Mercer-Steffensen inequality. For more information concerning these inequalities, one may refer to the in book [9], and the papers in [1], [11], [12] and [13].

In 1883, studying convex functions, Hermite (see [5]) has attained the important inequality. In 1893, not knowing Hermite's result, Hadamard (see [4]) has gotten its first part, the left inequality in equation (1.4).

Classic form of the Hermite-Hadamard inequality. Let $[a, b] \subset \mathbb{R}$ be a bounded closed interval.

Then every convex function $f:[a, b] \rightarrow \mathbb{R}$ satisfies the double inequality

$$
f\left(\frac{a+b}{2}\right) \leq \frac{1}{b-a} \int_{a}^{b} f(x) d x \leq \frac{f(a)+f(b)}{2} .
$$

For information as regards the Hermite-Hadamard inequality, one may refer to book [3], and the papers in [2], [8], [10] and [14].

\subsection{Set Barycenter in Euclidean Space}

The notion of the set barycenter is important for inequalities involving integrals.

Let $\mathcal{A} \subseteq \mathbb{R}^{k}$ be a set, let $\mu$ be a positive measure on $\mathbb{R}^{k}$ such that $\mu(\mathcal{A})>0$, and let $g: \mathcal{A} \rightarrow \mathbb{R}$ be an integrable function.

The barycenter of the set $\mathcal{A}$ respecting the measure $\mu$ is the point

$$
\left(\frac{1}{\mu(\mathcal{A})} \int_{\mathcal{A}} x_{1} d \mu, \ldots, \frac{1}{\mu(\mathcal{A})} \int_{\mathcal{A}} x_{k} d \mu\right)
$$

belonging to the convex hull of the set $\mathcal{A}$.

The integral mean of the function $g$ respecting the measure $\mu$ is the number

$$
\frac{1}{\mu(\mathcal{A})} \int_{\mathcal{A}} g\left(x_{1}, \ldots, x_{k}\right) d \mu
$$

belonging to the convex hull of the set $g(\mathcal{A})$.

Let $g_{1}, \ldots, g_{k}: \mathcal{A} \rightarrow \mathbb{R}$ be integrable functions. Let $\mathcal{C} \subseteq \mathbb{R}^{k}$ be a convex set containing the image set $\left\{\left(g_{1}(x), \ldots, g_{k}(x)\right): x \in \mathcal{A}\right\}$. Then every convex function $f: \mathcal{C} \rightarrow \mathbb{R}$, such that $f\left(g_{1}, \ldots, g_{k}\right)$ is integrable, satisfies the inequality

$$
f\left(\frac{1}{\mu(\mathcal{A})} \int_{\mathcal{A}} g_{1} d \mu, \ldots, \frac{1}{\mu(\mathcal{A})} \int_{\mathcal{A}} g_{k} d \mu\right) \leq \frac{1}{\mu(\mathcal{A})} \int_{\mathcal{A}} f\left(g_{1}, \ldots, g_{k}\right) d \mu
$$

The above inequality presents the integral form of the Jensen inequality for convex functions of several variables. If the function $f$ is affine, then the equality holds in equation (1.7). The same is true for equations (1.2), (1.3) and (1.4).

\section{Geometric Presentation of Inequalities}

Let $\mathcal{I} \subseteq \mathbb{R}$ be an interval, let $f: \mathcal{I} \rightarrow \mathbb{R}$ be a convex function, and let $c=\sum_{i=1}^{n} \alpha_{i} a_{i}$ be a convex combination of points $a_{i} \in \mathcal{I}$. To explain the Jensen inequality visually, we take the convex combination $C=\sum_{i=1}^{n} \alpha_{i} A_{i}$ of the points $A_{i}=\left(a_{i}, f\left(a_{i}\right)\right)$ belonging to the graph of $f$. The center $c$ is in the convex hull of line points $a_{i}$,

$$
c \in \operatorname{conv}\left\{a_{1}, \ldots, a_{n}\right\},
$$




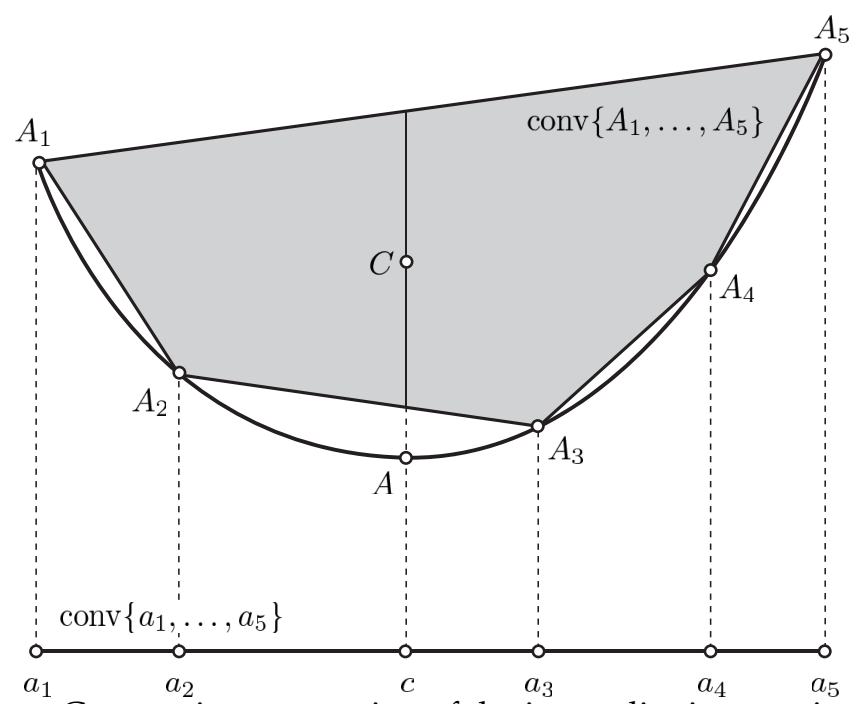

Figure 1. Geometric presentation of the inequality in equation (1.2)

as well as the center $C$ is in the convex hull of plane points $A_{i}$,

$$
C \in \operatorname{conv}\left\{A_{1}, \ldots, A_{n}\right\} .
$$

The convex hull in equation (2.2) is the convex polygon inscribed in the function epigraph. So, the polygon point

$$
C=\left(\sum_{i=1}^{n} \alpha_{i} a_{i}, \sum_{i=1}^{n} \alpha_{i} f\left(a_{i}\right)\right)=\left(c, \sum_{i=1}^{n} \alpha_{i} f\left(a_{i}\right)\right)
$$

is above the graph point

$$
A=(c, f(c))=\left(c, f\left(\sum_{i=1}^{n} \alpha_{i} a_{i}\right)\right),
$$

which can be seen in Figure 1. Therefore, the relation between ordinates of points $A$ and $C$,

$$
f\left(\sum_{i=1}^{n} \alpha_{i} a_{i}\right) \leq \sum_{i=1}^{n} \alpha_{i} f\left(a_{i}\right)
$$

represents the classical discrete form of the Jensen inequality for convex functions of one variable.

The visual explanation of the Hermite-Hadamard inequality for a convex function $f:[a, b] \rightarrow \mathbb{R}$ depends on two lines. We use the secant line function $f_{\{a, b\}}^{\mathrm{sec}}$ whose graph passes through the points $(a, f(a))$ and $(b, f(b))$, and for $c \in(a, b)$ a supporting line function $f_{\{c\}}^{\text {sup }}$ whose graph passes through the point $(c, f(c))$.

So we get the curvilinear trapezoid which contains the supporting trapeze, and which is contained in the bounding trapeze. This is clearly seen in Figure 2. By calculating the areas of all three shapes, it follows that

$$
(b-a) f_{\{c\}}^{\mathrm{sup}}\left(\frac{a+b}{2}\right) \leq \int_{a}^{b} f(x) d x \leq(b-a) f_{\{a, b\}}^{\mathrm{sec}}\left(\frac{a+b}{2}\right) .
$$

We could also obtain the above result by integrating the double inequality

$$
f_{\{c\}}^{\text {sup }}(x) \leq f(x) \leq f_{\{a, b\}}^{\mathrm{sec}}(x)
$$

over the interval $[a, b]$.

Applying the formula $f_{\{c\}}^{\text {sup }}(c)=f(c)$ to the midpoint $c=(a+b) / 2$ to the supporting line function (the supporting trapeze area attains a maximum value at the midpoint), we get

$$
f_{\left\{\frac{a+b}{2}\right\}}^{\text {sup }}\left(\frac{a+b}{2}\right)=f\left(\frac{a+b}{2}\right) .
$$




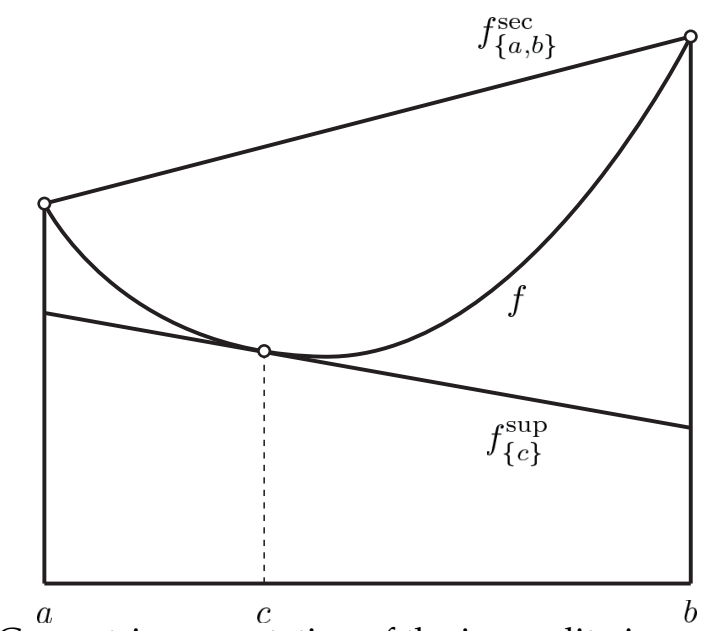

Figure 2. Geometric presentation of the inequality in equation (2.5)

Using the affinity of the secant line function, we obtain

$$
f_{\{a, b\}}^{\mathrm{sec}}\left(\frac{a+b}{2}\right)=\frac{1}{2} f_{\{a, b\}}^{\mathrm{sec}}(a)+\frac{1}{2} f_{\{a, b\}}^{\mathrm{sec}}(b)=\frac{f(a)+f(b)}{2} .
$$

Involving the above equalities to equation (2.5) and dividing with $b-a$, we achieve the classical form of the Hermite-Hadamard inequality,

$$
f\left(\frac{a+b}{2}\right) \leq \frac{1}{b-a} \int_{a}^{b} f(x) d x \leq \frac{f(a)+f(b)}{2} .
$$

\section{Preceding Inequality for Functions of One Variable}

The aim of this section is give the basic inequality from which can be derived both the Jensen and HermiteHadamard inequality.

Through the section we will use a bounded closed interval $[a, b] \subset \mathbb{R}$ with endpoints $a<b$, and a convex function $f:[a, b] \rightarrow \mathbb{R}$.

Every number $x \in \mathbb{R}$ can be presented by the unique affine combination

$$
x=\frac{b-x}{b-a} a+\frac{x-a}{b-a} b,
$$

which is convex if and only if the number $x$ belongs to the interval $[a, b]$. Applying the affinity of $f_{\{a, b\}}^{\text {sec }}$ to the equality in (3.1), we obtain the secant line equation

$$
f_{\{a, b\}}^{\mathrm{sec}}(x)=\frac{b-x}{b-a} f(a)+\frac{x-a}{b-a} f(b) .
$$

The function $f$ has both left and right derivative on $(a, b)$. Given a point $c \in(a, b)$ and a slope $\kappa \in\left[f^{\prime}(c-), f^{\prime}(c+)\right]$, we get the supporting line equation

$$
f_{\{c\}}^{\text {sup }}(x)=\kappa(x-c)+f(c) .
$$

Finally, we have the double inequality

$$
f_{\{c\}}^{\mathrm{sup}}(x) \leq f(x) \leq f_{\{a, b\}}^{\mathrm{sec}}(x), x \in[a, b] .
$$

Theorem 3.1. Let $[a, b] \subset \mathbb{R}$ be a bounded closed interval, let $\sum_{i=1}^{n} \alpha_{i} a_{i}$ be a convex combination of points $a_{i} \in[a, b]$, and let $\alpha a+\beta b$ be the unique convex combination of endpoints $a$ and $b$ such that

$$
\alpha a+\beta b=\sum_{i=1}^{n} \alpha_{i} a_{i} .
$$


Then every convex function $f:[a, b] \rightarrow \mathbb{R}$ satisfies the double inequality

$$
f(\alpha a+\beta b) \leq \sum_{i=1}^{n} \alpha_{i} f\left(a_{i}\right) \leq \alpha f(a)+\beta f(b) .
$$

Proof. We put $c=\sum_{i=1}^{n} \alpha_{i} a_{i}$. Since $c \in[a, b]$, we have two cases depending on the position of the center $c$. (3.6).

If $c$ is the endpoint, $c \in\{a, b\}$, then the trivial inequality $f(c) \leq f(c) \leq f(c)$ represents the inequality in equation

If $c$ is the interior point, $c \in(a, b)$, then we use a supporting function $f_{\{c\}}^{\text {sup }}$ and the secant function $f_{\{a, b\}}^{\text {sec }}$ to obtain the series of inequalities

$$
\begin{aligned}
f(\alpha a+\beta b) & =f_{\{c\}}^{\mathrm{sup}}(\alpha a+\beta b)=\sum_{i=1}^{n} \alpha_{i} f_{\{c\}}^{\mathrm{sup}}\left(a_{i}\right) \\
& \leq \sum_{i=1}^{n} \alpha_{i} f\left(a_{i}\right) \\
& \leq \sum_{i=1}^{n} \alpha_{i} f_{\{a, b\}}^{\mathrm{sec}}\left(a_{i}\right)=f_{\{a, b\}}^{\mathrm{sec}}(\alpha a+\beta b) \\
& =\alpha f(a)+\beta f(b)
\end{aligned}
$$

including the double inequality in equation (3.6).

According to representing equation (3.1), the coefficients $\alpha$ and $\beta$ in Theorem 3.1 are determined with fractions

$$
\alpha=\frac{b-c}{b-a}, \beta=\frac{c-a}{b-a} .
$$

Corollary 3.1. Let $[a, b] \subset \mathbb{R}$ be a bounded closed interval, let $\mu$ be a positive measure on $\mathbb{R}$ such that $\mu([a, b])>0$, and let $\alpha a+\beta b$ be the unique convex combination of endpoints $a$ and $b$ such that

$$
\alpha a+\beta b=\frac{1}{\mu([a, b])} \int_{[a, b]} x d \mu .
$$

Then every convex function $f:[a, b] \rightarrow \mathbb{R}$ satisfies the double inequality

$$
f(\alpha a+\beta b) \leq \frac{1}{\mu([a, b])} \int_{[a, b]} f(x) d \mu \leq \alpha f(a)+\beta f(b) .
$$

Proof. We take the barycenter $c=\int_{[a, b]} x d \mu / \mu([a, b])$, and since $c \in[a, b]$, we have the following two cases.

If the measure $\mu$ is concentrated in the endpoint, $c \in\{a, b\}$, then we have the trivial inequality $f(c) \leq f(c) \leq f(c)$.

If the measure $\mu$ is not concentrated in the endpoint, $c \in(a, b)$, then we can use the framework of equation (3.7). Inserting the integral mean of $f$, and the equalities

$$
h(\alpha a+\beta b)=\frac{1}{\mu([a, b])} \int_{[a, b]} h(x) d \mu
$$

with $h=f_{\{c\}}^{\text {sup }}$ and $h=f_{\{a, b\}}^{\text {sec }}$ into equation (3.7), we obtain equation (3.10).

The inequalities in equations (3.6) and (3.10) cover the following.

Remark 3.1. The left inequality of equation (3.6) with $\sum_{i=1}^{n} \alpha_{i} a_{i}$ instead of the endpoints combination $\alpha a+\beta b$ presents the discrete form of the Jensen inequality in equation (1.2), concerning the interval $[a, b]$.

The left inequality of equation (3.10) with $\int_{[a, b]} x d \mu / \mu([a, b])$ instead of $\alpha a+\beta b$ presents the integral form of the Jensen inequality in equation (1.3), concerning the identity function $g(x)=x$ on the interval $[a, b]$.

Using the Riemann integral in Corollary 3.1, the condition in equation (3.9) gives the midpoint

$$
\frac{1}{2} a+\frac{1}{2} b=\frac{1}{b-a} \int_{a}^{b} x d x
$$

and its use in equation (3.10) implies the classic form of the Hermite-Hadamard inequality for convex functions of one variable,

$$
f\left(\frac{1}{2} a+\frac{1}{2} b\right) \leq \frac{1}{b-a} \int_{a}^{b} f(x) d x \leq \frac{1}{2} f(a)+\frac{1}{2} f(b) .
$$




\section{Generalization to Functions of Several Variables}

To generalize the inequalities of Section 3, we will use the smallest convex sets such as segments in the line and triangles in the plane. This kind of sets are called simplexes or simplices.

Let $S_{1}, \ldots, S_{k+1} \in \mathbb{R}^{k}$ be points. Their convex hull

$$
\mathcal{S}=\operatorname{conv}\left\{S_{1}, \ldots, S_{k+1}\right\}
$$

is the $k$-simplex in the space $\mathbb{R}^{k}$ if the points $S_{1}-S_{k+1}, \ldots, S_{k}-S_{k+1}$ are linearly independent. The points $S_{j}$ are called simplex vertices.

Through the section we will use a $k$-simplex $\mathcal{S} \subset \mathbb{R}^{k}$ with vertices $S_{1}, \ldots, S_{k+1}$, and a convex function $f: \mathcal{S} \rightarrow \mathbb{R}$. The interior of $\mathcal{S}$ will be denoted by $\mathcal{S}^{o}$.

Every point $P \in \mathbb{R}^{k}$ can be presented by the unique affine combination

$$
P=\sum_{j=1}^{k+1} \lambda_{j} S_{j},
$$

which is convex if and only if the point $P$ belongs to the $k$-simplex $\mathcal{S}$. The coefficients $\lambda_{j}$ can be calculated by using determinants presented in [11]. We will use the hyperplane passing through the graph points $\left(S_{j}, f\left(S_{j}\right)\right)$, and a supporting hyperplane passing through the graph point $(C, f(C))$ where $C \in \mathcal{S}^{o}$. Using denotation $f_{\left\{S_{1}, \ldots, S_{k+1}\right\}}^{\text {hyperplane }}$ for the hyperplane function, and $f_{\{C\}}^{\text {sup }}$ for the supporting hyperplane function, we have the double inequality

$$
f_{\{C\}}^{\text {sup }}(P) \leq f(P) \leq f_{\left\{S_{1}, \ldots, S_{k+1}\right\}}^{\text {hyperplane }}(P), P \in \mathcal{S} .
$$

Theorem 4.1. Let $\mathcal{S}=\operatorname{conv}\left\{S_{1}, \ldots, S_{k+1}\right\}$ be a $k$-simplex in the space $\mathbb{R}^{k}$, let $\sum_{i=1}^{n} \alpha_{i} A_{i}$ be a convex combination of points $A_{i} \in \mathcal{S}$, and let $\sum_{j=1}^{k+1} \sigma_{j} S_{j}$ be the unique convex combination of simplex vertices $S_{j}$ such that

$$
\sum_{j=1}^{k+1} \sigma_{j} S_{j}=\sum_{i=1}^{n} \alpha_{i} A_{i} .
$$

Then every convex function $f: \mathcal{S} \rightarrow \mathbb{R}$ satisfies the double inequality

$$
f\left(\sum_{j=1}^{k+1} \sigma_{j} S_{j}\right) \leq \sum_{i=1}^{n} \alpha_{i} f\left(A_{i}\right) \leq \sum_{j=1}^{k+1} \sigma_{j} f\left(S_{j}\right) .
$$

Proof. We put $C=\sum_{i=1}^{n} \alpha_{i} A_{i}$. Since $C \in \mathcal{S}$, we have three cases depending on the position of the center $C$.

If $C$ is the simplex vertex, $C \in\left\{S_{1}, \ldots, S_{k+1}\right\}$, then the trivial inequality $f(C) \leq f(C) \leq f(C)$ represents the inequality in equation (4.4).

If $C$ is the interior point of $\mathcal{S}, C \in \mathcal{S}^{\circ}$, then following the proof of Theorem 3.1, we get

$$
\begin{aligned}
f\left(\sum_{j=1}^{k+1} \sigma_{j} S_{j}\right) & =f_{\{C\}}^{\text {sup }}\left(\sum_{j=1}^{k+1} \sigma_{j} S_{j}\right)=\sum_{i=1}^{n} \alpha_{i} f_{\{C\}}^{\text {sup }}\left(A_{i}\right) \\
& \leq \sum_{i=1}^{n} \alpha_{i} f\left(A_{i}\right) \\
& \leq \sum_{i=1}^{n} \alpha_{i} f_{\left\{S_{1}, \ldots, S_{k+1}\right\}}^{\text {hyperplane }}\left(A_{i}\right)=f_{\left\{S_{1}, \ldots, S_{k+1}\right\}}^{\text {hyperplane }}\left(\sum_{j=1}^{k+1} \sigma_{j} S_{j}\right) \\
& =\sum_{j=1}^{k+1} \sigma_{j} f\left(S_{j}\right),
\end{aligned}
$$

which proves the double inequality in equation (4.4).

If $C$ is the interior point of the $l$-face $\mathcal{F}$ in the subspace $\mathbb{R}^{l}$ where $1 \leq l \leq k-1, C \in \mathcal{F}^{o}$, then we can apply the previous procedure to the $l$-simplex $\mathcal{F}$. If the face $\mathcal{F}=\operatorname{conv}\left\{S_{1}, \ldots, S_{l+1}\right\}$, then the coefficients $\sigma_{1}, \ldots, \sigma_{l+1}$ are positive, and the coefficients $\sigma_{l+2}, \ldots, \sigma_{k+1}$ are equal to zero. 
Corollary 4.1. Let $\mathcal{S}=\operatorname{conv}\left\{S_{1}, \ldots, S_{k+1}\right\}$ be a $k$-simplex in the space $\mathbb{R}^{k}$, let $\mu$ be a positive measure on $\mathbb{R}^{k}$ such that $\mu(\mathcal{S})>0$, and let $\sum_{j=1}^{k+1} \sigma_{j} S_{j}$ be the unique convex combination of simplex vertices $S_{j}$ such that

$$
\sum_{j=1}^{k+1} \sigma_{j} S_{j}=\left(\frac{1}{\mu(\mathcal{S})} \int_{\mathcal{S}} x_{1} d \mu, \ldots, \frac{1}{\mu(\mathcal{S})} \int_{\mathcal{S}} x_{k} d \mu\right)
$$

Then every convex function $f: \mathcal{S} \rightarrow \mathbb{R}$ satisfies the double inequality

$$
f\left(\sum_{j=1}^{k+1} \sigma_{j} S_{j}\right) \leq \frac{1}{\mu(\mathcal{S})} \int_{\mathcal{S}} f\left(x_{1}, \ldots, x_{k}\right) d \mu \leq \sum_{j=1}^{k+1} \sigma_{j} f\left(S_{j}\right) .
$$

Proof. We can apply the procedure of proving Corollary 3.1 by utilizing the framework of the proof of Theorem 4.1.

Using the inequalities in equations (4.4) and (4.7), we can obtain the following.

Remark 4.1. The left inequality of equation (4.4) with $\sum_{i=1}^{n} \alpha_{i} A_{i}$ instead of the vertices combination $\sum_{j=1}^{k+1} \sigma_{j} S_{j}$ presents the discrete form of the Jensen inequality in equation (1.2), concerning the simplex $\mathcal{S}$.

The left inequality of equation (4.7) with $\left(\int_{\mathcal{S}} x_{1} d \mu / \mu(\mathcal{S}), \ldots, \int_{\mathcal{S}} x_{k} d \mu / \mu(\mathcal{S})\right)$ instead of $\sum_{j=1}^{k+1} \sigma_{j} S_{j}$ gives a simple version of the integral form of the Jensen inequality for convex functions of $k$ variables in equation (1.7), concerning the simplex $\mathcal{S}$.

Using the Riemann integral in Corollary 4.1, the condition in equation (4.6) gives the barycentric point

$$
\frac{1}{k+1} \sum_{j=1}^{k+1} S_{j}=\left(\frac{1}{\operatorname{vol}(\mathcal{S})} \int_{\mathcal{S}} x_{1} d x_{1} \ldots d x_{k}, \ldots, \frac{1}{\operatorname{vol}(\mathcal{S})} \int_{\mathcal{S}} x_{k} d x_{1} \ldots d x_{k}\right),
$$

and its use in equation (4.7) gives the Hermite-Hadamard inequality for convex functions of $k$ variables,

$$
f\left(\frac{1}{k+1} \sum_{j=1}^{k+1} S_{j}\right) \leq \frac{1}{\operatorname{vol}(\mathcal{S})} \int_{\mathcal{S}} f\left(x_{1}, \ldots, x_{k}\right) d x_{1} \ldots d x_{k} \leq \frac{1}{k+1} \sum_{j=1}^{k+1} f\left(S_{j}\right) .
$$

\section{Acknowledgements}

The author would like to thank Velimir Pavić who has graphically prepared Figure 1 and Figure 2.

\section{References}

[1] Bjelica, M., Refinement and converse of Brunk-Olkin inequality. Journal of Mathematical Analysis and Applications 272 (1998), 462-467.

[2] Chen, F., A Note on Hermite-Hadamard inequalities for products of convex functions. Journal of Applied Mathematics 2013 (2013), Article ID 935020.

[3] Dragomir, S. S. and Pearce, Ch. E. M., Selected Topics on Hermite-Hadamard Inequalities and Applications. RGMIA Monographs. Victoria University, Melbourne, AU, 2000.

[4] Hadamard, J., Étude sur les propriétés des fonctions entières et en particulier d'une fonction considerée par Riemann. Journal de Mathématiques Pures et Appliquées 58 (1893), 171-215.

[5] Hermite, Ch., Sur deux limites d'une intégrale définie. Mathesis 3 (1883), 82.

[6] Jensen, J. L. W. V., Om konvekse Funktioner og Uligheder mellem Middelværdier. Nyt tidsskrift for matematik. B. 16 (1905), 49-68.

[7] Jensen, J. L. W. V., Sur les fonctions convexes et les inégalités entre les valeurs moyennes. Acta Mathematica 30 (1906), 175-193. 
[8] Lyu, S. L., On the Hermite-Hadamard inequality for convex functions of two variables. Numerical Algebra, Control and Optimization 4 (2014), 1-8.

[9] Niculescu, C. P. and Persson, L. E., Convex Functions and Their Applications. Canadian Mathematical Society. Springer, New York, USA, 2006.

[10] Niculescu, C. P. and Persson, L. E., Old and new on the Hermite-Hadamard inequality. Real Analysis Exchange 29 (2003), 663-685.

[11] Pavić, Z., Generalizations of Jensen-Mercer's inequality. Journal of Pure and Applied Mathematics: Advances and Applications 11 (2014), 19-36.

[12] Pavić, Z., Extension of Jensen's inequality to affine combinations. Journal of Inequalities and Applications 2014 (2014), Article 298.

[13] Pečarić, J. E., A simple proof of the Jensen-Steffensen inequality. American Mathematical Monthly 91 (1984), 195-196.

[14] Wang, J., Li, X., Fečkan, M. and Zhou, Y., Hermite-Hadamard-type inequalities for Riemann-Liouville fractional integrals via two kinds of convexity. Applicable Analysis 92 (2013), 2241-2253.

\section{Affiliations}

ZlatKo PAVIĆ

AdDRESS: University of Osijek, Mechanical Engineering Faculty in Slavonski Brod, 35000, Slavonski Brod-Croatia. E-MAIL: Zlatko.Pavic@sfsb.hr 